\title{
Approximate Inverse Preconditioning in the Parallel Solution of Sparse Eigenproblems
}

\author{
Luca Bergamaschi, Giorgio Pini \\ Dipartimento di Metodi e Modelli Matematici per le Scienze Applicate \\ Università di Padova, via Belzoni 7, 35131 Padova, Italy
}

Flavio Sartoretto

Dipartimento di Informatica

Università di Venezia, Via Torino 155, 30171 Mestre VE, Italy

\begin{abstract}
A preconditioned scheme for solving sparse symmetric eigenproblems is proposed. The solution strategy relies upon the DACG algorithm, which is a Preconditioned Conjugate Gradient algorithm for minimizing the Rayleigh Quotient. A comparison with the well established ARPACK code, shows that when a small number of the leftmost eigenpairs is to be computed, DACG is more efficient than ARPACK. Effective convergence acceleration of DACG is shown to be performed by a suitable approximate inverse preconditioner (AINV). The performance of such a preconditioner is shown to be safe, i.e. not highly dependent on a drop tolerance parameter. On sequential machines, AINV preconditioning proves a practicable alternative to the effective incomplete Cholesky factorization, and is more efficient than Block Jacobi. Due to its parallelizability, the AINV preconditioner is exploited for a parallel implementation of the DACG algorithm. Numerical tests account for the high degree of parallelization attainable on a Cray T3E machine and confirm the satisfactory scalability properties of the algorithm. A final comparison with PARPACK shows the (relative) higher efficiency of AINV-DACG.
\end{abstract}

KEY WORDS generalized eigenproblem, sparse approximate inverse, parallel algorithm

\section{Introduction}

The computation of a small number of the leftmost eigenpairs (the smallest eigenvalues and corresponding eigenvectors) of the problem $A \boldsymbol{x}=\lambda B \boldsymbol{x}$, where $A$ and $B$ are large, sparse, symmetric positive definite matrices, is an important task in many scientific applications. Typical examples are the vibrational analysis of mechanical structures [1], 
the lightwave technology [35], and the spectral superposition approach for the solution of large sets of first order linear differential equations [13]. Several techniques for solving the generalized eigenproblem have been proposed: subspace iteration [2,27], Lanczos method [12,21,26], and, more recently, restarted Arnoldi-Lanczos algorithm [22], Jacobi-Davidson method [34], and optimization methods by Conjugate Gradient (CG) schemes [16,32].

In this paper an optimization method, called DACG (Deflation-Accelerated Conjugate Gradient) [17], is exploited. DACG computes sequentially the eigenpairs by CG minimizations of the Rayleigh quotient over subspaces of decreasing size. In a previous work [7] the asymptotic convergence rate of DACG, when preconditioned with $A^{-1}$, has been proven to be proportional to the square root of the relative separation of the eigenvalue being sought and the next higher one. Such a convergence rate compares well with that of the efficient $\mathrm{CG}$ scheme for the solution of linear systems [7]. When DACG is preconditioned with the inverse of the incomplete Cholesky decomposition of $A$ (called IC(0)), quite the same convergence speed as using $A^{-1}$ is obtained, at a lower computational cost, thus yielding a very effective method [6]. Its convergence behavior is similar to that of the algorithm proposed in [33].

To evaluate the efficiency of DACG, we performed a numerical comparison between DACG and the Implicitly Restarted Arnoldi/Lanczos Method, as implemented in the well established ARPACK package [23].

Recently, a class of preconditioners, known as the approximate inverse preconditioners, have been extensively studied by many authors in order to accelerate, in a parallel environment, iterative methods for solving linear systems $A \boldsymbol{x}=\boldsymbol{b}$. These preconditioners explicitly compute an approximation, say $M$, to $A^{-1}$. The application of the preconditioner needs only matrix vector products, which is more easily parallelized than solving two triangular systems, as in the IC $(0)$ preconditioner. The first class of approximate inverse preconditioners proposed was based on the minimization of the Frobenius norm $\|I-M A\|_{F}$. Among these methods we mention SPAI, proposed in [19]. Modifications to SPAI, attempting to reduce its high cost, were devised in [11], where the so called self preconditioned MR algorithm is proposed. The FSAI [20] and the AINV technique [4], are approximate inverse techniques based on the sparse factorization of $A^{-1}$. In [5] it is shown that preconditioning by a product of triangular factors performs better than other techniques, mainly because the fill-in of the preconditioner is reduced. Note that only the AINV and FSAI techniques preserve the positive definiteness of the problem, which is essential in our application. The construction of the FSAI preconditioner seems to be easily parallelizable than AINV. Recently, however, an efficient parallel construction of AINV, based upon graph partitioning, has been proposed [3]. The FSAI algorithm requires an a priori sparsity pattern of the approximate factor, which is not easy to provide in unstructured sparse problems. On the other hand, AINV is based upon a drop tolerance, which is more convenient for our problems. In this paper we restrict our attention to the AINV technique, numerical experiments with FSAI being in progress [8].

The high performance yielded by the AINV-CG technique in the solution of linear systems, suggests the idea of using AINV also for preconditioning the eigenvalue problem. The choice of an effective preconditioner is a matter of experience. Our paper is intended to give a contribution for guiding the user in solving large, sparse eigenproblems. We have exploited the sequential AINV-DACG algorithm for the solution of Finite Element (FE), Mixed Finite Element (MFE), and Finite Difference (FD) eigenproblems, both in two and three dimensions. In the computation of the AINV preconditioner, a drop tolerance param- 
eter, $\varepsilon$, which determines the fill-in of the triangular factor, has to be selected. Numerical results prove that the overall CPU time is usually not much influenced by this parameter. The sequential simulations show that the number of iterations of AINV-DACG is slightly larger than for IC(0)-DACG, and an increase in the CPU time is recorded. On the other hand, the IC(0) preconditioner requires the solution of two triangular linear systems. These intrinsically sequential operations ruin the parallel performance of the IC(0)-DACG algorithm. Thus, the lower sequential efficiency of AINV-DACG is scored acceptable, in view of a parallelization, since AINV needs only two highly parallelizable, matrix-vector products.

We followed the research direction pioneered in $[28,29,30]$, where several preconditioning techniques where exploited in the parallelization of eigensolution schemes, based upon the Rayleigh quotient minimization. A parallel implementation of the AINV-DACG algorithm has been performed via a data-parallel approach. Ad-hoc data-distribution techniques allow for reducing the amount of communication among the processors, which could spoil the parallel performance of the ensuing code. Numerical tests on a Cray T3E Supercomputer show the high degree of parallelism attainable by the code, together with its good scalability level.

This paper is organized as follows: $\S 2$ sketches the sequential DACG algorithm. $\S 3$ describes the AINV-DACG approach, presents the numerical results obtained by exploiting the sequential code with various preconditioners, and a numerical comparison with ARPACK package. In $\S 4$ the parallelization strategy is described, and the numerical results obtained by running the parallel code are reported, together with a comparison with PARPACK, the parallel version of ARPACK. The conclusions are drawn in $\S 5$.

\section{DACG algorithm}

We deal with the computation of the $s$ smallest eigenpairs of the eigenproblem

$$
A \boldsymbol{x}=\lambda B \boldsymbol{x}
$$

$A, B$ being two sparse, symmetric, positive definite (SPD), $N \times N$ matrices. The real positive eigenvalues and corresponding eigenvectors are denoted by $\lambda_{1} \leq \lambda_{2} \leq \ldots \leq \lambda_{N}$, and $\boldsymbol{u}_{1}, \boldsymbol{u}_{2}, \ldots, \boldsymbol{u}_{N}$, respectively. The DACG algorithm sequentially computes the eigenpairs, starting from the leftmost one $\left(\lambda_{1}, \boldsymbol{u}_{1}\right)$. To evaluate the $j$-th eigenpair, $j>1$, DACG minimizes the Rayleigh quotient in a subspace which is $B$-orthogonal to the $j-1$ eigenvectors previously computed. More precisely, computing $\lambda_{j}$ requires the minimization of

$$
q(\boldsymbol{z})=\frac{\boldsymbol{z}^{T} A \boldsymbol{z}}{\boldsymbol{z}^{T} B \boldsymbol{z}}
$$

where

$$
\boldsymbol{z}=\boldsymbol{x}-U_{j}\left(U_{j}^{T} B \boldsymbol{x}\right), \quad U_{j}=\left[\boldsymbol{u}_{1}, \ldots, \boldsymbol{u}_{j-1}\right], \boldsymbol{x} \in \mathbb{R}^{N} .
$$

To obtain $\left(\lambda_{1}, \boldsymbol{u}_{1}\right)$, DACG minimizes the Rayleigh quotient (2.2) with $\boldsymbol{z}=\boldsymbol{x}\left(U_{1}=\emptyset\right)$. The $s$ leftmost eigenpairs are computed by the following Conjugate Gradient procedure. 
DO $j=1, s$

1. Start with an initial vector $\boldsymbol{x}_{0}$ such that $U_{j}^{T} B \boldsymbol{x}_{0}=0$, set $k=0, \beta_{0}=0$. Choose tolerances $\varepsilon_{1}, \varepsilon_{2}$.

2. Compute $\boldsymbol{x}_{0}^{\prime}=A \boldsymbol{x}_{0}, \quad \boldsymbol{x}_{0}^{\prime \prime}=B \boldsymbol{x}_{0}, \quad q_{0} \equiv q\left(\boldsymbol{x}_{0}\right)=\boldsymbol{x}_{0}^{T} \boldsymbol{x}_{0}^{\prime} / \boldsymbol{x}_{0}^{T} \boldsymbol{x}_{0}^{\prime \prime}$

3. REPEAT

(a) $\boldsymbol{g}_{k} \equiv \nabla q\left(\boldsymbol{x}_{k}\right)=\frac{2}{\boldsymbol{x}_{k}^{T} \boldsymbol{x}_{k}^{\prime \prime}}\left[\boldsymbol{x}_{k}^{\prime}-q_{k} \boldsymbol{x}_{k}^{\prime \prime}\right]$.

(b) $\boldsymbol{g}_{k}^{\prime}=\boldsymbol{M g}_{k}, \quad M$ SPD preconditioner

(c) IF $k>0$ THEN $\beta_{k}=\frac{\boldsymbol{g}_{k}^{T}\left(\boldsymbol{g}_{k}^{\prime}-\boldsymbol{g}_{k-1}^{\prime}\right)}{\boldsymbol{g}_{k-1}^{T} \boldsymbol{g}_{k-1}^{\prime}}$,

(d) $\tilde{\boldsymbol{p}}_{k}=\boldsymbol{g}_{k}^{\prime}+\beta_{k} \boldsymbol{p}_{k-1}$

(e) $\boldsymbol{p}_{k}=\tilde{\boldsymbol{p}}_{k}-\sum_{i=1}^{j-1}\left(\boldsymbol{v}_{i}^{T} \tilde{\boldsymbol{p}}_{k}\right) \boldsymbol{u}_{i}$

(f) $\boldsymbol{p}_{k}^{\prime}=A \boldsymbol{p}_{k}, \quad \boldsymbol{p}_{k}^{\prime \prime}=B \boldsymbol{p}_{k}$.

(g) $\alpha_{k}=\underset{t}{\operatorname{argmin}}\left\{q\left(\boldsymbol{x}_{k}+t \boldsymbol{p}_{k}\right)\right\}$

(h) $\boldsymbol{x}_{k+1}=\boldsymbol{x}_{k}+\alpha_{k} \boldsymbol{p}_{k}, \quad \boldsymbol{x}_{k+1}^{\prime}=\boldsymbol{x}_{k}^{\prime}+\alpha_{k} \boldsymbol{p}_{k}^{\prime}, \quad \boldsymbol{x}_{k+1}^{\prime \prime}=\boldsymbol{x}_{k}^{\prime \prime}+\alpha_{k} \boldsymbol{p}_{k}^{\prime \prime}$.

(i) $q_{k+1} \equiv q\left(\boldsymbol{x}_{k+1}\right)=\boldsymbol{x}_{k+1}^{T} \boldsymbol{x}_{k+1}^{\prime} / \boldsymbol{x}_{k+1}^{T} \boldsymbol{x}_{k+1}^{\prime \prime}$

(j) $k=k+1$

UNTIL $\quad \frac{\left|q_{k}-q_{k-1}\right|}{q_{k}}<\epsilon_{1} \quad$ or $\quad \frac{\left\|\boldsymbol{x}_{k}^{\prime}-q_{k} \boldsymbol{x}_{k}^{\prime \prime}\right\|_{2}}{\left\|\boldsymbol{x}_{k}^{\prime}\right\|_{2}}<\epsilon_{2}$.

4. $\lambda_{j}=q_{k}, \quad \boldsymbol{u}_{j}=\boldsymbol{x}_{k} / \sqrt{\boldsymbol{x}_{k}^{T} \boldsymbol{x}_{k}^{\prime \prime}}, \quad \boldsymbol{v}_{j}=B \boldsymbol{u}_{j}$

END DO

\section{Remarks.}

- The coefficient $\alpha_{k}$ in (g) is computed as $\alpha_{k}=\frac{n d-m b+\sqrt{\Delta}}{2(b c-a d)}$, with $a=\boldsymbol{p}_{k}^{T} \boldsymbol{x}_{k}^{\prime}, \quad b=\boldsymbol{p}_{k}^{T} \boldsymbol{p}_{k}^{\prime}, \quad c=\boldsymbol{p}_{k}^{T} \boldsymbol{x}_{k}^{\prime \prime}, \quad d=\boldsymbol{p}_{k}^{T} \boldsymbol{p}_{k}^{\prime \prime}, \quad m=\boldsymbol{x}_{k}^{T} \boldsymbol{x}_{k}^{\prime}, \quad n=\boldsymbol{x}_{k}^{T} \boldsymbol{x}_{k}^{\prime \prime}$, $\Delta=(n d-m b)^{2}-4(b c-a d)(m a-n c)$.

- $q_{k+1}$ in step (i) is evaluated without performing any scalar products as:

$$
q_{k+1}=\frac{m+2 a \alpha_{k}+b \alpha_{k}^{2}}{n+2 c \alpha_{k}+d \alpha_{k}^{2}}
$$

- The computational cost of a single iteration is mainly determined by three steps: (i) the application of the preconditioner $\boldsymbol{M} \boldsymbol{g}_{k}$, (ii) two matrix-vector products $A \boldsymbol{p}_{k}, B \boldsymbol{p}_{k}$ and (iii) the re-orthogonalization procedure (step (e) of the algorithm). The first two blocks of operations are independent of the eigenpair level, while the complexity of the third one linearly increases with $j$.

In [7] an asymptotic convergence analysis of DACG is performed, assuming that $M \equiv$ $A^{-1}$, i.e. the product $\boldsymbol{y}=A^{-1} \boldsymbol{g}_{k}$ is computed as a preconditioned conjugate gradient solution of the linear system $A \boldsymbol{y}=\boldsymbol{g}_{k}$. The asymptotic convergence rate of DACG is proven to be proportional to the square root of the relative separation between the desired eigenvalue and the next higher one. Using $A^{-1}$ as the preconditioner makes each iteration computationally expensive, however numerical results of FE sample tests [6] show 
that preconditioning with the cheaper incomplete Cholesky preconditioner IC $(0)$, yields comparable convergence rates and provides a very cost-effective algorithm.

\section{Sequential AINV-DACG algorithm}

This section is devoted to analyze the sequential performance, in the eigenanalysis, of the approximate inverse preconditioner AINV, which was developed in [4] for linear systems.

\subsection{The AINV preconditioner}

Following [4], we now shortly recall the main steps for the construction of the AINV preconditioner. Given a symmetric positive definite matrix $A$ it is possible to explicitly construct $A^{-1}$ by a conjugate Gram-Schmidt process applied to an arbitrary set of linearly independent vectors. If one chooses the canonical basis $\left(\boldsymbol{e}_{1}, \ldots \boldsymbol{e}_{N}\right)$, this process produces a unit upper triangular matrix $\tilde{Z}$, and a diagonal matrix $D$ such that

$$
A^{-1}=\tilde{Z} D^{-1} \tilde{Z}^{T} \text {. }
$$

Actually, even with sparse $A$, the factor $\tilde{Z}$ is usually dense. The AINV preconditioner is based on the idea of building a sparse approximation to $\tilde{Z}$ by reducing the amount of fill-in occurring in the computation. This is obtained by annihilating either all the elements outside selected positions in $\tilde{Z}$ or those whose absolute value is smaller than a drop tolerance $\varepsilon$. We selected this latter strategy, which is suitable for dealing with unstructured sparse matrices.

If this process is completed successfully, which is guaranteed for example when $A$ is an H-matrix (see [4] for a proof), a unit upper triangular matrix $\bar{Z}$ and a diagonal matrix with positive diagonal entries $\bar{D}$, are obtained. Thus, a sparse approximate inverse of $A$, $M=\bar{Z} \bar{D}^{-1} \bar{Z}^{T}=Z Z^{T}$ is produced, being $Z=\bar{Z} D^{-1 / 2}$.

\begin{tabular}{|l|l|r|r|}
\hline pb. & type & $N$ & $N_{n z}$ \\
\hline 1 & 3d - FE & 4,560 & 64,030 \\
2 & 2d-FE & 7,744 & 53,952 \\
3 & 2d-FE & 10,593 & 72,738 \\
4 & 2d-MFE $(B=I)$ & 28,600 & 152,204 \\
5 & 3d - FE $(B=I)$ & 42,189 & 602,085 \\
6 & 3d-FD $(B=I)$ & 64,000 & 438,400 \\
7 & 3d - FE $(B=I)$ & 80,711 & 325,835 \\
8 & 3d - FD $(B=I)$ & 216,000 & $1,490,400$ \\
\hline
\end{tabular}

Table 1. Main characteristics of our sample problems. $N=$ matrix size, $N_{n z}=$ nonzero elements in matrix $A ; \mathrm{FE}=$ Finite Elements, $\mathrm{FD}=$ Finite Differences, MFE $=$ Mixed Finite Elements. 


\subsection{Numerical tests}

We now report the numerical results obtained applying the DACG procedure to eight FE, MFE and FD problems with large sizes.

All CPU times are measured in seconds.

Table 1 lists the main features of the test problems, which arise from the discretization of parabolic equations in two and three space dimensions. Problems 2 and 3 come from the solution of 2-D groundwater flow equations by FE [15]. Problem 4 is the result of MFE discretization of the 2-D Richard's equation [9]. Problems 1 and 5 arise from FE discretization of 3-D flow equations [14]. Problem 7 arises from FE discretization of 3D groundwater flow in a multiaquifer [18]. Problems 6 and 8 come from a standard 3D Laplacean discretization applying 7-point centered FD. Sequential experiments were performed on an Alpha Station 600Mhz with $512 \mathrm{Mb}$ RAM. We compared the AINV preconditioner with $\mathrm{IC}(0)$ and the Jacobi preconditioner. We set $\epsilon_{1}=10^{-8}, \epsilon_{2}=10^{-3}$.
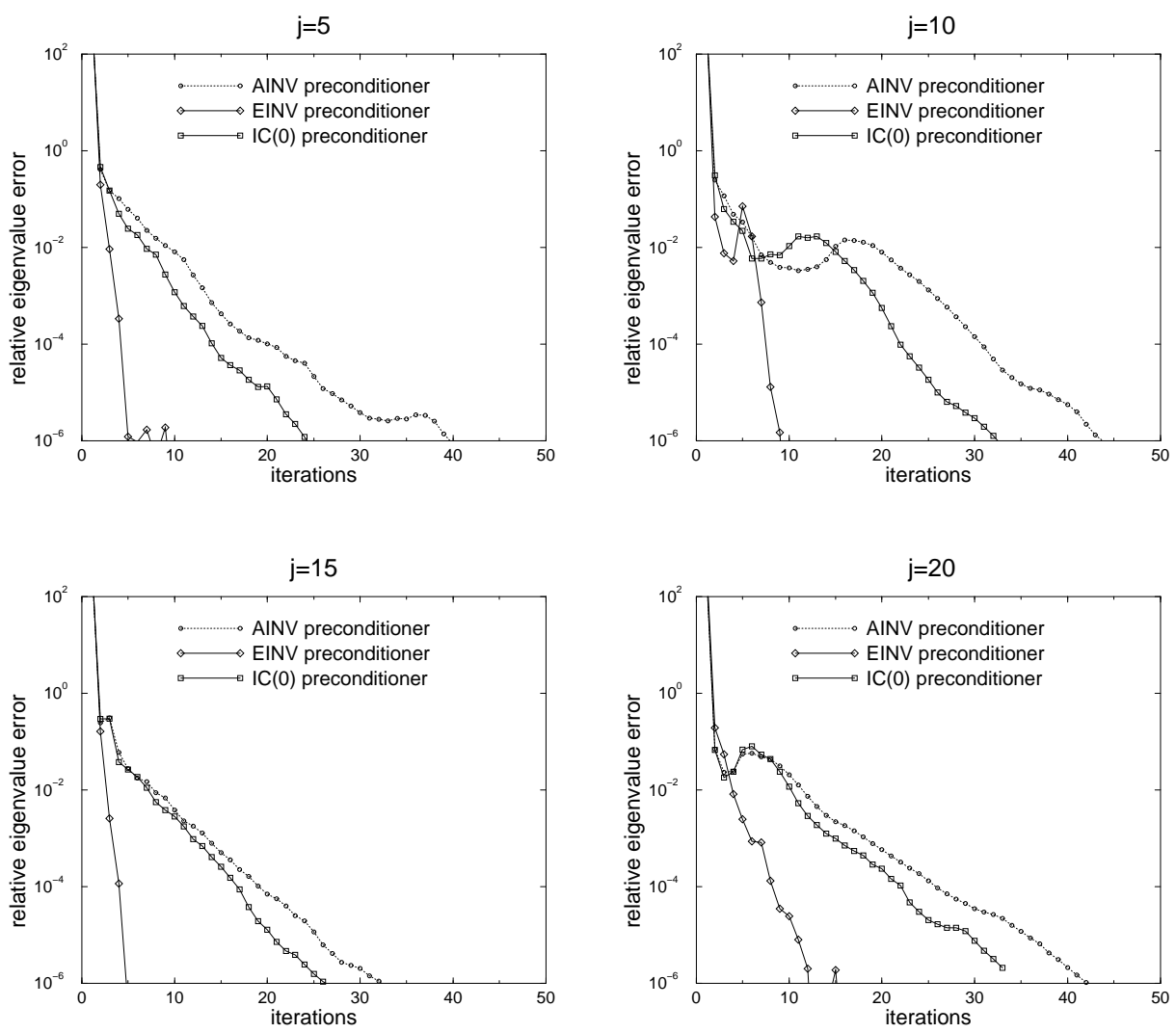

Figure 1. Convergence history of DACG when preconditioned with $\operatorname{AINV}(0.05), \operatorname{IC}(0)$ and $A^{-1}$ (EINV - Exact Inverse). Computation of $\left(\lambda_{j}, \boldsymbol{u}_{j}\right), j=5,10,15,20$ for problem $1(N=4560)$.

The construction of the AINV preconditioner depends on the drop tolerance parameter $\varepsilon$ which drives the amount of fill-in in the factor $Z$. Table 2 gives the total number 


\begin{tabular}{|r|r|rr|r|rr|}
\hline & \multicolumn{3}{|c|}{$N=4,560$} & \multicolumn{3}{c|}{$N=28,600$} \\
\hline$\varepsilon$ & \multicolumn{1}{|c|}{$N_{n z}^{(Z)}$} & \# iter. & CPU(s) & $N_{n z}^{(Z)}$ & \# iter. & CPU(s) \\
\hline 0.01 & 143,790 & 262 & 5.19 & $2,047,982$ & 950 & 233.69 \\
0.05 & 36,234 & 449 & 4.59 & 464,705 & 1614 & 143.77 \\
0.10 & 23,269 & 752 & 6.48 & 201,765 & 2276 & 141.82 \\
0.25 & 14,793 & 1100 & 8.61 & 133,100 & 4109 & 197.92 \\
\hline
\end{tabular}

Table 2. Number of iterations and CPU seconds for four values of the drop tolerance $\varepsilon$, in the computation of $s=10$ eigenpairs on problems 1 and $4 . N_{n z}^{(Z)}$ is the number of nonzero entries in the factor $Z$.

of iterations and overall CPU time for some choices of $\varepsilon$ in the computation of $s=10$ eigenpairs for problems 1 and 4, which are representative of the entire set. Note that the overall computing time does not highly depend on $\varepsilon$. We selected the value of the drop tolerance yielding about the same sparsity percentage as IC $(0)$. We found that two values $\varepsilon=0.1$, or $\varepsilon=0.05$ were sufficient to meet this criterion. However, this strategy yielded slow convergence for problems 3,4 and 7, thus $\varepsilon$ was chosen in order to get a number of iterations approximately equal to that obtained with the $\operatorname{IC}(0)$ preconditioner. We finally set $\varepsilon=0.1$ for problems $2,4,6,7,8 ; \varepsilon=0.05$ for the remaining problems. The code has been run on the 8 test problems in the computation of $s=10$ and $s=40$ eigenpairs. Recall that the cost of the re-orthogonalization step increases with $s$, thus the performance of the preconditioned algorithm changes with $s$. Figure 1 shows the convergence history of preconditioned DACG for problem 1, when computing a selected number of the leftmost eigenpairs. Solving the other problems, we get similar convergence profiles. Note that on the average the convergence rates obtained using either the IC $(0)$ or the AINV preconditioner are the same. The convergence profiles of these two preconditioners are given together with that of $A^{-1}$, which yields the optimal convergence rate.

Table 3 reports the overall number of iterations, the total CPU time, and the number of nonzero entries in the preconditioning factor. Note that AINV-DACG is on the average slightly worst than IC(0)-DACG, both in terms of number of iterations and CPU time. When computing 40 eigenpairs, in the most favorable case AINV-DACG is 1.04 times slower than IC(0)-DACG (problem 5) and 1.79 times slower (problem 7) in the worst one. Moreover in all test problems AINV-DACG requires a higher number of iterations than IC(0)-DACG, with the only exception of problem $3, s=40$. The CPU time $\mathrm{T}(M)$, spent for the computation of the AINV preconditioner turns out to be negligible when compared to the overall computing time, as can be inferred from the last column of Table 3 . When computing $s=40$ eigenpairs, $T(M)$ is at most $1 \%$ of the total CPU time (test problem 1 ).

The Jacobi-DACG algorithm yields a much higher number of iterations than IC(0)DACG and AINV-DACG. The total time of Jacobi-DACG can be more than 60 times higher (problem 7, $s=10$ ) when compared to IC(0)-DACG. The diagonal scaling, however, should not be discarded in view of a parallel implementation, since it has the highest parallelizability potential.

In problems 4,6 , and 8 the CPU times needed by Jacobi-DACG are not much larger than using AINV. For this reason we have made a further study on the performance of the Block-Jacobi preconditioner. Let Jacobi $(k)$ mean that the size of the block is $k$. In Table 4 


\begin{tabular}{|c|c|c|c|c|c|c|c|}
\hline \multirow[b]{2}{*}{$\mathrm{pb}$} & \multirow[b]{2}{*}{$N_{n z}^{(M)}$} & \multirow[b]{2}{*}{ precond } & \multicolumn{2}{|c|}{$s=10$} & \multicolumn{2}{|c|}{$s=40$} & \multirow[b]{2}{*}{$\mathrm{T}(M)$} \\
\hline & & & iter & $\mathrm{CPU}$ & iter & $\mathrm{CPU}$ & \\
\hline \multirow[t]{3}{*}{1} & 34,295 & $\mathrm{IC}(0)$ & 290 & 3.05 & 1,392 & 21.31 & 0.01 \\
\hline & 36,234 & AINV (0.05) & 449 & 4.59 & 1,910 & 28.40 & 0.25 \\
\hline & 4,560 & Jacobi & 5,004 & 33.19 & 16,570 & 179.33 & - \\
\hline \multirow[t]{3}{*}{2} & 30,848 & $\mathrm{IC}(0)$ & 994 & 13.97 & 3,175 & 71.40 & 0.01 \\
\hline & 34,503 & AINV (0.1) & 1,066 & 14.74 & 3,768 & 83.92 & 0.21 \\
\hline & 7,744 & Jacobi & 2,791 & 28.63 & 8,452 & 154.54 & - \\
\hline \multirow[t]{3}{*}{3} & 41,665 & $\mathrm{IC}(0)$ & 1,285 & 26.64 & 5,684 & 194.30 & 0.01 \\
\hline & 97,051 & AINV (0.05) & 1,407 & 35.07 & 5,659 & 218.22 & 0.26 \\
\hline & 10,593 & Jacobi & 3,334 & 51.43 & 18,009 & 502.70 & - \\
\hline \multirow[t]{3}{*}{4} & 85,402 & $\mathrm{IC}(0)$ & 1,978 & 102.93 & 8,153 & 687.49 & 0.02 \\
\hline & 201,765 & $\operatorname{AINV}(0.1)$ & 2,276 & 141.82 & 9,474 & 906.93 & 0.43 \\
\hline & 28,600 & Jacobi & 7,537 & 274.21 & 30,361 & 2211.53 & - \\
\hline \multirow[t]{3}{*}{5} & 322,137 & $\mathrm{IC}(0)$ & 913 & 109.55 & 4,421 & 818.25 & 0.11 \\
\hline & 341,523 & $\operatorname{AINV}(0.05)$ & 1,024 & 127.56 & 4,792 & 853.27 & 0.58 \\
\hline & 42,189 & Jacobi & 2,942 & 222.94 & 13,950 & 1922.16 & - \\
\hline \multirow[t]{3}{*}{6} & 251,200 & $\mathrm{IC}(0)$ & 751 & 120.73 & 3,583 & 862.10 & 0.07 \\
\hline & 251,200 & $\operatorname{AINV}(0.1)$ & 1,145 & 156.39 & 5,212 & 1200.79 & 0.69 \\
\hline & 64,000 & Jacobi & 2,026 & 218.49 & 11,502 & 2248.22 & - \\
\hline \multirow[t]{3}{*}{7} & 203,273 & $\mathrm{IC}(0)$ & 1,203 & 194.33 & 4,944 & 1402.95 & 0.09 \\
\hline & $1,640,603$ & $\operatorname{AINV}(0.1)$ & 1,288 & 437.88 & 5,428 & 2510.61 & 2.21 \\
\hline & 80,711 & Jacobi & 96,899 & 12531.31 & * & $*$ & - \\
\hline \multirow[t]{3}{*}{8} & 853,200 & $\mathrm{IC}(0)$ & 1,133 & 692.41 & 5,355 & 5565.70 & 0.26 \\
\hline & 853,200 & AINV (0.1) & 1,672 & 966.92 & 8,023 & 7411.38 & 1.94 \\
\hline & 216,000 & Jacobi & 3,329 & 1517.63 & 17,908 & 13687.09 & - \\
\hline
\end{tabular}

Table 3. Performance of IC(0), AINV and Jacobi preconditioners as applied to the computation of $s=10$ and $s=40$ leftmost eigenpairs of the seven sample problems. The last column, T $(M)$, reports the time spent for constructing the preconditioner; $N_{n z}^{(M)}$ is the number of nonzero entries in the preconditioner factor. An asterisk means no convergence attained.

we report the results of $\operatorname{Jacobi}(k)$-DACG $(k=1,2,3)$ on the five largest test problems.

When $k \geq 4$ the growing cost of a single iteration is not balanced by the (slight) decrease in the iteration number. Hence the total CPU time is larger on all the test problems and it is not reported in Table 4. Note that $k=3$ allows for the smallest CPU time in four of the five problems considered. On problems 4, 6 and 8, that arise from FD (or MFE) discretization, the CPU times spent with Jacobi(3) are close to those obtained using AINV (cf. Table 3). On these problems, Block-Jacobi performance becomes even closer to that of AINV in parallel runs, as documented in Section 4. On FE problems 5 and 7 the time with Jacobi(3) is much larger than the one with AINV, particularly for problem 7, where the ratio between the two computing times is larger than 20 (compare Tables 3 and 4). If $s=40$ eigenpairs are to be computed, the cost of a single iteration depends essentially on the orthogonalization process. Hence the $\operatorname{Jacobi}(k)$ preconditioner, always requiring an higher number of iterations than AINV, is in this case much less competitive. 


\begin{tabular}{|c|rr|rr|rr|}
\hline & \multicolumn{2}{|c|}{$k=1$} & \multicolumn{2}{c|}{$k=2$} & \multicolumn{2}{c|}{$k=3$} \\
pb & iter. & CPU & iter. & CPU & iter. & CPU \\
\hline 4 & 7537 & 274.21 & 6486 & 248.90 & 6101 & 231.31 \\
5 & 2942 & 222.94 & 2577 & 203.39 & 2530 & 199.89 \\
6 & 2026 & 218.49 & 1594 & 178.38 & 1422 & 162.59 \\
7 & 96899 & 12531.31 & 88756 & 11975.74 & 71813 & 9437.95 \\
8 & 3329 & 1517.63 & 2118 & 968.32 & 2280 & 1050.97 \\
\hline
\end{tabular}

Table 4. Performance of Jacobi $(k)$ preconditioners, $k=1,2,3$, in the DACG computation of the $s=10$ leftmost eigenpairs of the largest test problems.

\subsection{Comparison with ARPACK}

The efficiency of our DACG code has been compared with that of ARPACK [23], a standard package for computing a number of the eigenvalues and, on demand, the corresponding eigenvectors, of the generalized problem for arbitrary matrices. ARPACK is based upon the Implicitly Restarted Arnoldi Method (IRAM), a variant of the Arnoldi algorithm. When dealing with symmetric matrices, this method reduces to a variant of the Lanczos algorithm, called the Implicitly Restarted Lanczos Method.

This code has a reverse communication interface which needs a routine for performing sparse matrix-vector multiplications and, to compute a number of the smallest eigenvalues, a linear system solver. Note that the efficiency of ARPACK depends upon these routines, which must be supplied by the user. To perform an unbiased comparison, we exploited the most efficient routines for sparse computations that was available to us. We supplied a sparse linear system solver, based upon the Preconditioned Conjugate Gradient (PCG) algorithm, which efficiently evaluates the solution of symmetric, positive definite linear systems.

The computation of the leftmost $s$ eigenpairs by ARPACK requires the computation of $l$ Lanczos basis vectors, where $l>s$ is to be guessed by the user. Choosing $l$ for optimal efficiency is problem dependent. Usually, setting $l \geq 2 s$ is suggested. In our tests, we set $l=2 s$.

The precision in the computation of the eigenpairs is specified by the user via a tolerance $\tau$. This parameter must be carefully set by the user. The accuracy depends also on the tolerance, $\tau_{1}$, required for a suitable norm of the residual in the inner PCG solver.

Table 5 compares DACG efficiency with that of ARPACK, in the computation of the 10 smallest eigenpairs of our test problems. IC(0)-DACG is considered, and, accordingly, the PCG solver exploited by ARPACK is preconditioned via IC $(0)$. In order to get comparable accuracy in the computations, leaving $\epsilon_{1}=10^{-8}, \epsilon_{2}=10^{-3}$, we performed ARPACK computations choosing $\tau=10^{-8}, \tau_{1}=10^{-4}\left(\tau_{1}=10^{-6}\right.$ in problem 7). Table 5 reports the average relative errors and relative residuals, for both methods. The average residuals turn out to be comparable in DACG and ARPACK. Except when $N=7,744$ and $N=$ 80,711 , the average error is appreciably smaller in DACG, which is not surprising, since it computes the eigenvalues by direct minimization of the Rayleigh quotient, while ARPACK exploits a matrix whose eigenvalues approximate those of the given problem. Incidentally, we found that multiple eigenvalues are computed at a higher accuracy by DACG. On the other hand, inspecting Table 5, one can infer that the CPU time spent by ARPACK is larger 


\begin{tabular}{|r|r|rrr|rrr|}
\hline pb & $N$ & \multicolumn{3}{|c|}{ ARPACK } & \multicolumn{3}{c|}{ DACG } \\
& & avg error & avg resid & CPU & avg error & avg resid & CPU \\
\hline 1 & 4,560 & $9.88 \mathrm{e}-06$ & $5.59 \mathrm{e}-02$ & 10.12 & $5.97 \mathrm{e}-09$ & $8.72 \mathrm{e}-03$ & 3.05 \\
2 & 7,744 & $2.76 \mathrm{e}-06$ & $8.41 \mathrm{e}-03$ & 31.99 & $5.32 \mathrm{e}-06$ & $1.39 \mathrm{e}-02$ & 13.97 \\
3 & 10,593 & $1.17 \mathrm{e}-06$ & $6.20 \mathrm{e}-03$ & 44.71 & $2.88 \mathrm{e}-08$ & $4.36 \mathrm{e}-02$ & 26.64 \\
4 & 28,600 & $1.69 \mathrm{e}-06$ & $5.94 \mathrm{e}-03$ & 181.90 & $8.87 \mathrm{e}-08$ & $1.80 \mathrm{e}-03$ & 102.93 \\
5 & 42,189 & $5.66 \mathrm{e}-06$ & $1.89 \mathrm{e}-03$ & 266.90 & $1.34 \mathrm{e}-07$ & $6.24 \mathrm{e}-04$ & 109.55 \\
6 & 64,000 & $3.93 \mathrm{e}-06$ & $3.97 \mathrm{e}-04$ & 177.52 & $6.35 \mathrm{e}-08$ & $5.13 \mathrm{e}-04$ & 120.73 \\
7 & 80,711 & $7.55 \mathrm{e}-09$ & $4.34 \mathrm{e}-03$ & 500.41 & $3.14 \mathrm{e}-08$ & $2.40 \mathrm{e}-03$ & 194.33 \\
8 & 216,000 & $3.23 \mathrm{e}-06$ & $8.69 \mathrm{e}-04$ & 903.62 & $6.93 \mathrm{e}-08$ & $6.34 \mathrm{e}-04$ & 692.41 \\
\hline
\end{tabular}

Table 5. Comparison between ARPACK with IC(0)-preconditioned CG, and IC(0)-DACG, when computing $s=10$ eigenpairs.

than for DACG, being up to three times the latter (when $N=4,560$ ). Note that the time spent by ARPACK to solve problems 6 and 8 is closer, than in the other problems, to that spent by DACG. This result is likely to be ascribed to the nature of the eigenproblems, which come from Finite Difference discretizations, rather than Finite Element ones.

Using a lower stopping parameter for the inner CG solver inside ARPACK, produces far more accurate eigenpairs but drastically increases the CPU time. Setting, for example, $\tau_{1}=\tau=10^{-12}$, the computing time is from two to three times higher than with $\tau_{1}=$ $10^{-4}, \tau=10^{-8}$.

About the influence of the parameter $\tau$ in ARPACK, note that leaving $\tau_{1}=10^{-4}$, and setting for example $\tau=10^{-4}$, would provide higher residuals, up to an order of magnitude, and may erroneously lead to the computation of some $k$-th eigenvalue, $k>s=10$. On the other hand the CPU time spent by ARPACK can decrease at most $20 \%$, with respect to the case $\tau=10^{-8}$, anyhow remaining larger than that spent by DACG.

These results show that when computing a small number of eigenpairs is pursued, DACG is to be preferred over ARPACK. Computing a larger number $s>10$ of eigenpairs, the CPU time spent by DACG can be larger, due to the re-orthogonalization step, whose computational cost increases with $s$, resulting in a large portion of the overall computing time when the number of evaluated eigenpairs increases.

\section{Parallel AINV-DACG algorithm}

This Section presents the parallelization strategies developed for the AINV-DACG algorithm, describes their implementation and numerical testing.

\subsection{Algorithm parallelization}

Our AINV-DACG algorithm was parallelized via a data-parallel approach.

The DACG algorithm can be straightforwardly decomposed into a number of scalar products, daxpy-like linear combinations of vectors, $\alpha \boldsymbol{v}+\beta \boldsymbol{w}$, and matrix-vector multiplications. We focussed on parallelizing these tasks, assuming that the code is to be run 


\begin{tabular}{|l|l|l|l|l|}
\hline & \multicolumn{2}{|c|}{$A \boldsymbol{v}$} & \multicolumn{2}{c|}{$Z Z^{\boldsymbol{T}} \boldsymbol{v}$} \\
$i$ & $k$ & \# data & $k$ & \# data \\
\hline 1 & 2 & 2009 & 2 & 83 \\
2 & 1,3 & 2009,2009 & 1,3 & 83,198 \\
3 & 2,4 & 2009,2009 & 2,4 & 112,83 \\
4 & 3,5 & 2009,2009 & 3,5 & 83,85 \\
5 & 4,6 & 2009,2009 & 4,6 & 85,206 \\
6 & 5,7 & 2009,2009 & 5,7 & 207,83 \\
7 & 6,8 & 2009,2009 & 6,8 & 83,83 \\
8 & 7 & 2009 & 7 & 83 \\
\hline
\end{tabular}

Table 6. Number of floating point values (\#data) exchanged between processors $i$ and $k$, when performing the products $A \boldsymbol{v}$ and $Z Z^{T} \boldsymbol{v}$. (Problem 5, $N=42,189, p=8$ ).

on a machine with $p$ identical, powerful processors, our model machine being a Cray T3E Supercomputer.

Scalar products, $\boldsymbol{v} \cdot \boldsymbol{w}$, are distributed among the $p$ processors by a classical block mapping. Let $h=[N / p]$. Define the sets $R_{j}=\{h(j-1)+1, \ldots, h j\}$, for each $j=$ $1, \ldots, p-1$, together with $R_{p}=\{h(p-1)+1, \ldots, N\}$. For a given vector $\boldsymbol{z}$, let $\boldsymbol{z}_{(j)}=\left(\boldsymbol{z}^{(i)}, i \in R_{j}\right), j=1, \ldots, p$, be the sub-vector consisting of the contiguous elements in $\boldsymbol{z}$ whose indices fall in $\boldsymbol{R}_{\boldsymbol{j}}$. Our data-distribution chart requires that processor $j$, $j=1, \ldots, p$, computes the scalar product of the sub-vectors $\boldsymbol{v}_{(j)}, \boldsymbol{w}_{(j)}$. The results are combined via a global reduce operation.

For any vector combination $\boldsymbol{z}=\alpha \boldsymbol{v}+\beta \boldsymbol{w}$, our parallelization strategy requires that each processor $j$ computes only the sub-block $\boldsymbol{z}_{(j)}=\alpha \boldsymbol{v}_{(j)}+\beta \boldsymbol{w}_{(j)}$. One can see that quite in the whole DACG algorithm, broadcasting to the other processors $\boldsymbol{z}_{(j)}$ is not needed, which is a fair reduction in the amount of data exchange needed.

In each AINV-DACG iteration the vectors $\boldsymbol{y}=C \boldsymbol{v}$ have to be computed, when $C=$ $A, B, Z, Z^{T}$, respectively. Each $N \times N$ matrix $C$ is uniformly row-partitioned among the $p$ processors, so that processor $i$ handles the rows $r \in R_{i}$. Approximately $[N / p]$ rows of $A$, $B, Z$ and $Z^{T}$ are stored on each processor. Thus, large problems can be solved by using a small amount of memory, provided that a suitably large number of processors is activated.

The AINV preconditioning technique demands for a number of matrix-vector multiplications $Z Z^{T} \boldsymbol{v}, Z$ being an upper triangular matrix. Inside our parallel algorithm, the vector $\boldsymbol{w}=Z^{T} \boldsymbol{v}$ is first computed, then $\boldsymbol{z}=Z \boldsymbol{w}$ is evaluated. In principle, some memory saving can be attained by avoiding to store $Z^{T}$. To reduce data communication, however, we prefer to explicitly store $Z^{T}$. For the same reason, the whole A and B are stored, instead of their upper (or lower) triangular part.

The implementation of the matrix-vector product is tailored for application to sparse matrices, by minimizing data communication between processors [10]. Let $P^{i}=\left\{c_{r j} \neq\right.$ $\left.0, r \in R_{i}\right\}$ be the set including the nonzero elements belonging to processor $i$. Define $P_{1}^{i}=$ $\left\{c_{r j} \in P^{i}, j \notin R_{i}\right\}$. Let $C_{k}^{i}=\left\{j: c_{r j} \in P_{1}^{i}, j \in R_{k},\right\} ; G_{k}^{i}=\left\{r: c_{r j} \in P_{1}^{i}, j \in R_{k}\right\}$. Recall that processor $i$ has in its local memory the components $v^{(j)}, j \in R_{i}$. To complete the matrix-vector product it needs from processor $k$ precisely the elements $\boldsymbol{v}^{(j)}, j \in C_{k}^{i}$. At the same time it must send to processor $k$ that elements $\boldsymbol{v}^{(j)}, j \in G_{k}^{i}$. Our matrix-vector 
routine exchanges precisely these elements and no more, thus providing a large saving in the communication time. For instance, Table 6 displays the amount of data exchanged by each processor in the $A \boldsymbol{v}$ and the $Z Z^{T} \boldsymbol{v}$ matrix vector products, when solving problem 5 with 8 processors. In the trivial matrix-vector algorithm, a processor communicates with each other by exchanging in the selected case approximately $[N / p]=[42,189 / 8]=5273$ values. From Table 6 we can see that using our approach each processor sends/receives data to/from at most 2 other processors. Moreover, the amount of data exchanged is far smaller than $[N / p]$, especially when dealing with $Z$ and $Z^{T}$. Note that when $C=Z$, $G_{k}^{i}=\emptyset, \forall i<k$, whereas when $C=Z^{T}$, then $C_{k}^{i}=\emptyset, \forall i<k$.

\subsection{Code development}

The AINV-DACG algorithm was carefully implemented into a Fortran 77 code. The parallel tasks were accomplished by calls to the MPI library, release 1.2 [25]. Fortran 77 plus MPI lead to a highly portable code, which can be run on clusters of workstations, as well as dedicated parallel machines. The reduction operation needed for evaluating the scalar products in parallel is accomplished by calling the MP I ALLREDUCE routine.

All matrices were stored in CSR format [31].

\subsection{Numerical results}

Numerical experiments were performed by running our parallel code on the Cray T3E/1200 Supercomputer installed at the CINECA Consortium in Bologna, Italy. The machine is a stand alone system made by a set of DEC-Alpha 21164 processors, performing at a peak rate of $1200 \mathrm{Mflop} / \mathrm{s}$, which are interconnected by a 3D toroidal network having a $480 \mathrm{MByte} / \mathrm{s}$ payload bandwidth. Each processor, also called processing element $(\mathrm{PE})$, is equipped with its local memory, and it is directly connected to the communication net. In the present configuration there are 128 PEs with 256-MByte RAM. Beside a primary cache, each PE has an additional 96 KByte, 3-way set associative cache.

\begin{tabular}{|r||r|r|r|r|r|r||r|r|r|r|r|}
\hline pb. & $T_{1}$ & $T_{2}$ & $T_{4}$ & $T_{8}$ & $T_{16}$ & $T_{32}$ & $S_{2}$ & $S_{4}$ & $S_{8}$ & $S_{16}$ & $S_{32}$ \\
\hline 1 & 6.8 & 4.4 & 3.9 & 3.3 & 3.5 & 4.0 & 1.53 & 1.76 & 2.05 & 1.97 & 1.70 \\
2 & 19.2 & 13.8 & 9.7 & 7.4 & 8.7 & 13.9 & 1.40 & 1.98 & 2.60 & 2.20 & 1.38 \\
3 & 35.2 & 19.1 & 11.2 & 7.4 & 6.1 & 4.5 & 1.84 & 3.14 & 4.78 & 5.77 & 7.82 \\
4 & 122.7 & 66.6 & 37.0 & 21.5 & 13.6 & 10.6 & 1.84 & 3.32 & 5.70 & 9.05 & 11.54 \\
5 & 106.2 & 56.0 & 29.6 & 17.6 & 10.5 & 8.5 & 1.90 & 3.59 & 6.02 & 10.09 & 12.50 \\
6 & 133.6 & 69.1 & 38.0 & 21.5 & 13.3 & 9.3 & 1.93 & 3.51 & 6.20 & 10.04 & 14.39 \\
7 & 379.6 & 230.1 & 165.1 & 101.9 & 70.1 & 51.8 & 1.65 & 2.30 & 3.72 & 5.41 & 7.33 \\
8 & 644.7 & 339.4 & 176.4 & 95.2 & 53.5 & 31.5 & 1.90 & 3.66 & 6.77 & 12.06 & 20.47 \\
\hline
\end{tabular}

Table 7. AINV-DACG computation of $s=10$ eigenpairs. The elapsed time in seconds, $T_{p}$, together with the speed-up, $S_{p}$, recorded running on $p$ T3E processors are reported.

Table 7 shows the CPU time, $T_{p}$, spent by AINV-DACG for computing on $p$ T3E processors $s=10$ eigenpairs of our test problems.

Like for the sequential computations, our timings take care of the numerical core of the DACG code, i.e. the computations outlined in Section 2. The time needed for comput- 
ing the preconditioning matrix is not considered. As shown in Table 3, this is a negligible portion of the overall computing time, when $s \geq 10$ eigenpairs are evaluated. We are interested in measuring the parallelization degree of our algorithm, leaving to further analysis the problems raised by parallelizing the I/O operations. Thus, the CPU time consumed for data input is not considered. The time spent for computing pointer vectors for blockdistributing vectors and matrices (not needed in the sequential code), is not considered, too. The time spent for pointer evaluations is negligible with respect to that spent performing the core DACG algorithm, the former being in all our test cases less than $0.01 \%$ of the latter.

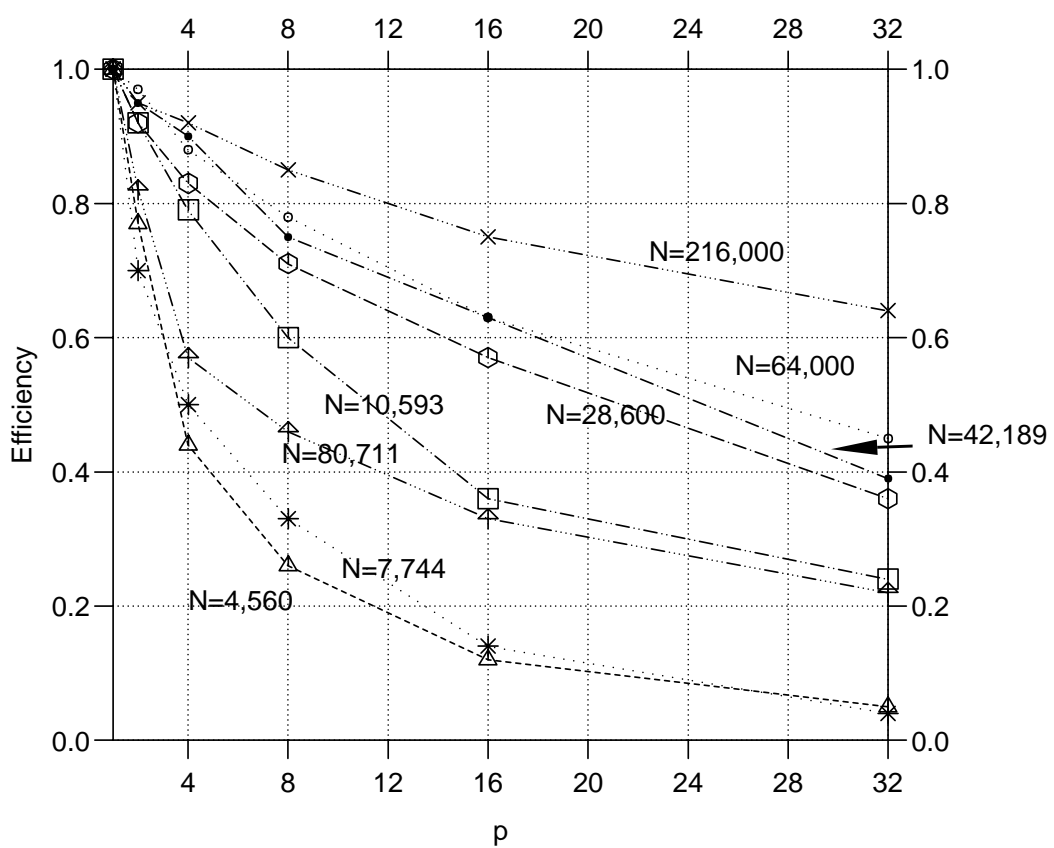

Figure 2. Efficiency of our parallel AINV-DACG code.

Columns 8-12 in Table 7 show the speed-ups recorded when computing $s=10$ eigenpairs on $2,4,8,16,32$ processors, respectively. Here, the speedup on $p$ processors is the ratio $S_{p}=T_{1} / T_{p}$ between the elapsed time spent running the parallel code on 1 and $p$ processors, respectively. Since we take into account merely the core algorithm, the additional computation done by the parallel code running on a single processor, over the sequential one, restricts to negligible pointer evaluations and (useless) MPI calls which immediately return. Our test problems are quite large, thus the overhead due to these calls is a negligible percentage of the overall CPU time. Eventually, recording the time spent running our parallel code on a single processor, we practically record the time spent by that equivalent segment of the sequential code, thus the speedup is properly defined.

Inspecting Table 7, we see that when $N<10,593$ (pb. 1 and 2) poor speedups are recorded. On the other hand, satisfactory values are reported in the larger test problems, leading up to $S_{p}=20.47$, when $p=32, N=216,000$ (pb. 8). For each given $p>4$, 
$N>7,744$, the speedup increases with the size of the problem (except when $N=80,711$, pb. 7, which will be treated in the sequel), showing that our approach is well tuned to largesize problems. Note that going from $N=7,744$ to $N=10,593$ a high increase in the speedup is observed, according to the higher fill-in experienced in the AINV preconditioner when switching from problem 2 to problem 3 (see Table 3 ).

Computing $s>10$ eigenpairs produces a better parallel performance, since the cost for re-orthogonalization becomes a larger part of the overall time spent by DACG. In the re-orthogonalization step most of the computation is carried out in parallel.

\begin{tabular}{|c|c|r|r|r|r|r|r|}
\hline & & \multicolumn{6}{|c|}{ problem 4} \\
\cline { 3 - 8 } & & 1 & 2 & 4 & 8 & 16 & 32 \\
\hline Jacobi & $T_{p}$ & 255.1 & 135.0 & 79.2 & 46.6 & 29.3 & 21.3 \\
$k=1$ & $S_{p}$ & - & 1.9 & 3.2 & 5.5 & 8.7 & 12.0 \\
& it & 7537 & 7537 & 7537 & 7537 & 7537 & 7537 \\
\hline Jacobi & $T_{p}$ & 237.4 & 124.4 & 69.9 & 40.1 & 24.5 & 17.0 \\
$k=2$ & $S_{p}$ & - & 1.9 & 3.4 & 5.9 & 9.7 & 14.0 \\
& it & 6486 & 6486 & 6486 & 6493 & 6653 & 6375 \\
\hline Jacobi & $T_{p}$ & 215.0 & 113.8 & 63.6 & 36.1 & 22.1 & 15.8 \\
$k=3$ & $S_{p}$ & - & 1.9 & 3.4 & 6.0 & 9.7 & 13.6 \\
& it & 6101 & 6127 & 6114 & 6113 & 6127 & 6121 \\
\hline AINV & $T_{p}$ & 122.7 & 66.6 & 37.0 & 21.5 & 13.6 & 10.6 \\
$\varepsilon=0.1$ & $S_{p}$ & - & 1.8 & 3.3 & 5.7 & 9.1 & 11.5 \\
& it & 2276 & 2276 & 2276 & 2276 & 2276 & 2276 \\
\hline & & \multicolumn{6}{|c|}{ problem 8} \\
& & \multicolumn{7}{|c|}{ number of processors $p$} \\
& & 1 & 2 & 4 & 8 & 16 & 32 \\
\hline Jacobi & $T_{p}$ & 855.8 & 496.6 & 250.3 & 136.0 & 71.5 & 42.9 \\
$k=1$ & $S_{p}$ & - & 1.7 & 3.4 & 6.3 & 12.0 & 19.9 \\
& it & 2919 & 3263 & 3185 & 3301 & 3171 & 3197 \\
\hline Jacobi & $T_{p}$ & 732.6 & 398.4 & 182.5 & 95.7 & 52.7 & 31.4 \\
$k=2$ & $S_{p}$ & - & 1.8 & 4.0 & 7.7 & 13.9 & 23.3 \\
& it & 2404 & 2404 & 2295 & 2310 & 2292 & 2314 \\
\hline Jacobi & $T_{p}$ & 683.3 & 307.6 & 158.1 & 99.4 & 51.5 & 26.5 \\
$k=3$ & $S_{p}$ & - & 2.2 & 4.3 & 6.9 & 13.3 & 25.8 \\
& it & 2287 & 2040 & 2035 & 2432 & 2273 & 2006 \\
\hline AINV & $T_{p}$ & 644.7 & 339.4 & 176.4 & 95.2 & 53.5 & 31.5 \\
$\varepsilon=0.1$ & $S_{p}$ & - & 1.9 & 3.7 & 6.8 & 12.1 & 20.5 \\
& it & 1672 & 1672 & 1672 & 1672 & 1672 & 1672 \\
\hline
\end{tabular}

Table 8. Computation of $s=10$ eigenpairs for problems 4 and 8, by Jacobi $(k)$-DACG and AINV-DACG. The elapsed time in seconds, $T_{p}$, the speed-up, $S_{p}$, and the number of iterations, it, performed running on $p$ T3E processors, are reported.

Figure 2 shows the efficiency of our code when the AINV preconditioning is exploited. The efficiency on $p$ processors is $E_{p}=T_{1} / p T_{p}=S_{p} / p$. Inspecting Figure 2 one can see that for a given number of processors, the efficiency usually increases with the size of the problem, when $N>7,744$. The best efficiency is achieved for $N=216,000$, showing the good scalability of our approach. 
Inspecting Table 7 and Figure 2 we see that the speedup and efficiency values obtained solving problem 7 (with $N=80,711$ ) are much lower than expected considering its relatively large size. This is due to the ill-conditioning of this problem (signaled also by the bad performance of the Jacobi preconditioning) which produces a $Z$ factor whose sparsity structure is not suitable to the uniform partitioning exploited in this work. Table 8 refers to solving test problems 4 and 8 via $\operatorname{Jacobi}(k), k=1,2,3$, and AINV preconditioning. Solving these problems, the Block-Jacobi preconditioner displayed close performances to AINV. As expected, the parallel Jacobi(3)-DACG and AINV-DACG require roughly the same computing time when a large number of processor is employed. On problem 8 , $p=2,4,16,32$, Jacobi(3), is even faster than AINV, thanks to its higher degree of parallelization.

Analyzing Table 8 note that the number of iterations performed using Jacobi $(k)$ preconditioning changes with the number of running processors, $p$. For example, when $N=$ 216,000 (pb. 8), $k=1$, the total number of iterations erratically changes with $p$, ranging from 2,919 to 3,301 . When $p=1$ the smallest number of iterations was performed, thus $S_{p}, p=2,4,8,16,32$, is smaller than if the number of iterations would be constant. This consideration can explain why for any $p$ the speedup is lower with Jacobi(1) than with AINV. This result is merely due to the change in the order of evaluation of the floating point operations, which occurs when the number of processors changes. Moreover, solving a given problem via Jacobi(1) running either the scalar code on our Alpha Station or the parallel one on a single T3E processor, can need different numbers of iterations (cf. problem 8, Tables 3 and 8 ). AINV preconditioning is numerically more robust, converging in the same number of iterations, irrespective of the number of running processors. This fact is interpreted as a further benefit of AINV over Jacobi $(k)$, when parallel codes are exploited.

\subsection{Comparison with PARPACK}

Now consider PARPACK [24], the parallelized version of ARPACK. The parallelization relies upon a message passing layer (we exploited MPI), and the reverse communication interface demands for a parallel matrix-vector routine together with a parallel linear system solver. We used the same efficient matrix-vector routine for sparse computations which was previously described, together with a parallel PCG solver, preconditioned by AINV.

\begin{tabular}{|r||r|r|r|r|r|r||r|r|r|r|r|}
\hline pb & $T_{1}$ & $T_{2}$ & $T_{4}$ & $T_{8}$ & $T_{16}$ & $T_{32}$ & $S_{2}$ & $S_{4}$ & $S_{8}$ & $S_{16}$ & $S_{32}$ \\
\hline 1 & 14.1 & 10.4 & 8.1 & 7.4 & 9.2 & 12.3 & 1.35 & 1.74 & 1.92 & 1.53 & 1.15 \\
2 & 45.3 & 36.2 & 30.2 & 24.5 & 32.7 & 46.3 & 1.25 & 1.50 & 1.85 & 1.38 & 0.98 \\
3 & 72.3 & 38.4 & 22.3 & 13.9 & 10.4 & 8.8 & 1.88 & 3.25 & 5.22 & 6.98 & 8.21 \\
4 & 245.8 & 128.7 & 71.0 & 41.5 & 27.9 & 23.4 & 1.91 & 3.46 & 5.92 & 8.82 & 10.49 \\
5 & 203.6 & 106.8 & 58.6 & 33.2 & 20.7 & 14.2 & 1.91 & 3.47 & 6.13 & 9.85 & 14.30 \\
6 & 161.8 & 81.2 & 45.1 & 25.9 & 17.1 & 11.8 & 1.99 & 3.58 & 6.25 & 9.47 & 13.76 \\
7 & 997.7 & 593.9 & 377.1 & 234.6 & 164.2 & 136.9 & 1.68 & 2.65 & 4.25 & 6.08 & 7.29 \\
8 & 787.7 & 397.7 & 209.8 & 114.3 & 66.7 & 43.1 & 1.98 & 3.75 & 6.89 & 11.81 & 18.26 \\
\hline
\end{tabular}

Table 9. CPU seconds, $T_{p}$, and speed-up, $S_{p}$, recorded running PARPACK on $p$ T3E processors. Computation of $s=10$ eigenpairs. 
Table 9 shows the CPU time and speedup recorded when computing by PARPACK the $s=10$ leftmost eigenpairs of our test problems. The values of the numerical parameters (number of Lanczos vectors, tolerances, etc.) are the same as in Section 3.3. Inspecting Tables 7 and 9 one can infer that the speedups are comparable with those of AINVDACG, which is not surprising, since DACG and our implementation of PARPACK share the matrix-vector routine. The remaining parts of both codes rely upon vector operations which are parallelized in the same manner.

On the other hand, comparing Tables 7 and 9 one can see that the CPU times spent by PARPACK are larger than those spent by our parallel AINV-DACG code. The conclusions drawn when comparing DACG with ARPACK apply also to the parallel codes.

\section{Conclusions}

An efficient parallel algorithm for computing the leftmost eigenpairs of large, sparse, symmetric, generalized eigenproblems has been devised, implemented and tested on a set of problems whose size ranges from 4,560 to 216,000 . The following points are worth noting.

- The sequential IC(0)-DACG code is more efficient than Lanczos method, as implemented in ARPACK package, when a small number of eigenpairs (say 10) is to be computed.

- The AINV preconditioner turns out to be a competitive alternative to $\mathrm{IC}(0)$ for the partial eigenvalue problem, in a sequential environment. Results on large size problems show that AINV-DACG requires from $4 \%$ to $80 \%$ CPU time more than IC(0)-DACG. In our parallel implementation, the Jacobi(3) preconditioner displays comparable performance with AINV on FD and MFE problems. On FE problems, and particularly on ill-conditioned ones, $\operatorname{Jacobi}(k)$ is not competitive with AINV.

- The pattern and convergence acceleration of the AINV preconditioner depend on a drop tolerance parameter, $\varepsilon$, which is problem dependent. This parameter has a strong impact on memory requirements, driving the fill-in of the preconditioner. However, the CPU time spent by AINV-DACG is usually not much influenced by the value of $\varepsilon$. Selecting $\varepsilon$ in $\{0.05,0.1\}$ proved sufficient to identify efficient preconditioners for all test problems.

- Careful parallel implementation of the AINV-DACG algorithm leads to an efficient code which recorded satisfactory speedup on a Cray T3E machine, running on $1 \leq p \leq 32$ processors. For $p=2,4,8,16,32$, the maximum speedups yielded are $S_{2}=1.93$, $S_{4}=3.66, S_{8}=6.77, S_{16}=12.06, S_{32}=20.47$.

- The code proves to be scalable on the Cray T3E. For a given number of processors, its efficiency increases with the size of the problem.

- A comparison with PARPACK, a parallelized version of ARPACK, shows the (relative) superiority of our parallelized AINV-DACG code. Their parallel efficiency is quite the same, but the CPU time consumed by PARPACK is larger, when a small number of eigenpairs is to be evaluated.

\section{Acknowledgements}

This work has been supported in part by the Italian MURST Project "Analisi Numerica: Metodi e Software Matematico", and CNR contract 98.01022.CT01. The first author has 
also been supported by EC contract \# IC15 CT96-211. Free accounting units on the T3E Supercomputer were given by CINECA, inside a frame research grant. We thank M. Benzi and $\mathrm{M}$. Tuma for providing the AINV code. We thank the referees for providing valuable suggestions.

\section{REFERENCES}

1. K. J. Bathe. Finite Element Procedures in Engineering Analysis. Prentice-Hall, Englewood Cliffs, 1982.

2. K. J. Bathe and E. Wilson. Solution methods for eigenvalue problems in structural dynamics. Int. J. Numer. Methods Eng., 6:213-226, 1973.

3. M. Benzi, J. Marin, and M. Tuma. A two-level parallel preconditioner based on sparse approximate inverses. In D. Kincaid et al., editor, Iterative Methods in Scientific Computing, pages 1-11. IMACS, 1998.

4. M. Benzi, C. D. Meyer, and M. Tüma. A sparse approximate inverse preconditioner for the conjugate gradient method. SIAM J. Sci. Comput., 17(5):1135-1149, 1996.

5. M. Benzi and M. Tüma. A comparative study of sparse approximate inverse preconditioners. Applied Numerical Mathematics, 30:305-340, 1999.

6. L. Bergamaschi, G. Gambolati, and G. Pini. Spectral analysis of large finite element problems by optimization methods. J. Shock and Vibr., 1(6):529-540, 1994.

7. L. Bergamaschi, G. Gambolati, and G. Pini. Asymptotic convergence of conjugate gradient methods for the partial symmetric eigenproblem. Numer. Lin. Alg. Appl., 4(2):69-84, 1997.

8. L. Bergamaschi, G. Pini, and F. Sartoretto. Factorized approximate inverse preconditioning of a sparse eigensolver. In proceedings of Parallel Computing 99, 2000 (in print).

9. L. Bergamaschi and M. Putti. Mixed finite elements and Newton-like linearization for the solution of Richard's equation. Int. J. Numer. Methods Eng., 45(8):1025-1046, 1999.

10. L. Bergamaschi and M. Putti. Efficient parallelization of preconditioned conjugate gradient schemes for matrices arising from discretizations of diffusion equations. In Proceedings of the Ninth SIAM Conference on Parallel Processing for Scientific Computing, March, 1999. (CD$\mathrm{ROM})$.

11. E. Chow and Y. Saad. Approximate inverse preconditioners via sparse-sparse iterations. SIAM J. Sci. Comput., 18(6):1657-1675, 1998.

12. T. Ericsson and A. Ruhe. The spectral transformation Lanczos method for the numerical solution of large sparse generalized symmetric eigenvalue problems. Math. Comp., 35:1251-1268, 1980.

13. G. Gambolati. On time integration of groundwater flow equations by spectral methods. Water Resour. Res., 29(4):1257-1267, 1993.

14. G. Gambolati, A. di Monaco, G. Galeati, F. Uliana, P. Mosca, and C. Mascardi. New approaches and applications in subsurface flow modeling: 3-D finite element analysis of dewatering for an electro-nuclear plant. In E. Custodio, editor, Groundwater Flow and Quality Modelling, pages 717-759. D. Reidel Publishing Company, 1988.

15. G. Gambolati, G. Pini, and M. Putti. Nested iterations for symmetric eigenproblems. SIAM J. Sci. Comput., 16(1):173-192, 1995.

16. G. Gambolati, G. Pini, and F. Sartoretto. An improved iterative optimization technique for the leftmost eigenpairs of large symmetric matrices. J. Comp. Phys., 74:41-60, 1988.

17. G. Gambolati, F. Sartoretto, and P. Florian. An orthogonal accelerated deflation technique for large symmetric eigenproblems. Comp. Methods App. Mech. Eng., 94:13-23, 1992.

18. G. Gambolati and P. Teatini. A block iterative finite element model for non-linear leaky aquifer systems. Water Resour. Res., 32(1):199-204, 1996.

19. M. J. Grote and T. Huckle. Parallel preconditioning with sparse approximate inverses. SIAM J. Sci. Comput., 18(3):838-853, 1997.

20. L. Yu. Kolotilina and A. Yu. Yeremin. Factorized sparse approximate inverse preconditioning I. Theory. SIAM J. Matrix Anal., 14:45-58, 1993.

21. C. Lanczos. An iteration method for the solution of the eigenvalue problem of linear differential 
and integral operators. J. Res. Nat. Bur. Standard, 45:255-282, 1950.

22. R. B. Lehoucq and D. C. Sorensen. Deflation techniques for an implicit restarted Arnoldi iteration. SIAM J. Matrix Anal., 17(4):789-821, 1996.

23. R. B. Lehoucq, D. C. Sorensen, and C. Yang. ARPACK Users Guide. Solution of Large Scale Eigenvalue Problem with Implicit Restarted Arnoldi Methods. SIAM, 1998.

24. K. J. Maschhoff and D. C. Sorensen. A portable implementation of ARPACK for distributed memory parallel architectures. In Proceedings of the Copper Mountain Conference on Iterative Methods, volume 1, April 9-13 1996.

25. P. Pacheco. Parallel Programming with MPI. Morgan Kaufmann, San Francisco, 1997.

26. C. C. Paige. Computational variants of the Lanczos method for the eigenproblem. J. Inst. Math. Applics., 10:373-381, 1972.

27. B. N. Parlett. The Symmetric Eigenvalue Problem. Prentice-Hall, Englewood Cliffs, NJ, 1980.

28. G. Pini. A parallel algorithm for the partial eigensolution of sparse symmetric matrices on the CRAY Y/MP. Parallel Computing, 17:553-562, 1991.

29. G. Pini and G. Gambolati. Parallel eigenanalysis for nested grids. In Proc. of 7th ACM Conference on Supercomputing, pages 281-287, Baltimore, 1993. ACM Press.

30. G. Pini and F. Sartoretto. Vector and parallel codes for large sparse eigenproblems. Supercomputer, 50:29-39, 1992.

31. Y. Saad. Iterative Methods for Sparse Linear Systems. PWS Publishing Company, Boston, MA, 1996.

32. F. Sartoretto, G. Pini, and G. Gambolati. Accelerated simultaneous iterations for large finite element eigenproblems. J. Comp. Phys., 81:53-69, 1989.

33. H. R. Schwarz. Eigenvalue problems and preconditioning. In Numerical Treatment of Eigenvalue Problems, volume 5, ISNM 96, pages 191-208. Birkhäuser, Basel, 1991.

34. H. A. Slejpen and H. A. van der Vorst. A Jacobi-Davidson method for linear eigenvalue problems. SIAM J. Matrix Anal., 17(2):401-425, 1996.

35. M. Zoboli and P. Bassi. The finite element method for anisotropic optical waveguides. In C. Someda and G. Stegeman, editors, Anisotropic and Nonlinear Optical Waveguides, pages 77-116, Amsterdam, 1992. Elsevier. 